\title{
Construcción de imagen y reputación en la mente de los consumidores
}

\section{Between Reason and Emotion. Building image and reputation in consumer minds}

Jaime Alberto Orozco Toro. Universidad Pontificia Bolivariana

Omar Muñoz Sánchez. Universidad de Santiago de Compostela

Recibido: 1-IV-2012 - Aceptado: 24-VII-2012

Resumen:

El presente artículo analiza la manera en que las empresas construyen imagen y reputación en la mente de sus stakeholders, a partir de procesos de comunicación estratégica. Debido a que persisten problemas para identificar las diferencias entre identidad, imagen y reputación, se realiza un estudio bibliográfico que permita dilucidar estas discrepancias conceptuales, para posteriormente identificar la forma en que los diferentes públicos perciben la comunicación empresarial y, cómo dichas percepciones se procesan en el cerebro de los consumidores. Se exploran también, la estructura de pensamiento de los stakeholders y, la forma en que se genera la conexión entre imagen-reputación-consumidor.

Palabras clave:

Imagen, reputación, percepción, pensamiento, marca

Abstract:

This article analyzes how firms build image and reputation in the minds of its stakeholders, from strategic communication processes. Due to persistent problems in identifying the differences between identity, image and reputation, a literature study is performed to elucidate these differences of opinion, and later identify how different audiences perceive business communication and how these perceptions are processed in the brains of consumers. It also explores the structure of thought of the stakeholders and how to build the connection between image-reputation-consumer.

Keywords:

Image, reputation, perception, thought, brand 


\section{Introducción}

Los encargados de la gestión empresarial de las grandes compañías están cada vez más interesados en conocer profundamente las percepciones que los stakeholders (grupos de interés y/o públicos objetivos) tienen de sus marcas; las investigaciones de mercados y los análisis de los procesos empresariales han ido incluyendo la forma en que los stakeholders reciben y procesan la información que las marcas les brindan a través de la publicidad y la comunicación corporativa. Sin embargo, persisten problemas estructurales significativos, como por ejemplo, entender la forma en que reaccionan los consumidores a ciertos tipos de publicidad, la manera en que las compañías deben construir su imagen (dicotomía entre la emotividad y la racionalidad), e incluso hasta llegar a aspectos tan esenciales como la confusión existente entre académicos y profesionales sobre los conceptos identidad, imagen y reputación, y la forma en que se construye imagen y reputación en la mente de los stakeholders.

Desde el ámbito académico el interés por conocer el funcionamiento del cerebro y sus reacciones a los estímulos de las marcas en la mente de los stakeholders no es nuevo. Lo primero que se debe tener en cuenta en esta relación cerebro mente es el hecho de saber que se trata una relación indisoluble, interdependiente y que no pueden ser contradictorias, por el contrario, se complementan y se necesitan mutuamente. Lo anterior obedece a que ambas forman parte del mismo individuo, "se trata de una vida humana" (Melendo, 2001: 129), la cual está constituida por una parte material y tangible, pero también conformada por una parte inmaterial, intangible y espiritual. Desde el ámbito empresarial, conocer cómo reaccionan los consumidores se ha convertido en una prioridad, sobre todo en medio de las circunstancias actuales: globalización de mercados, proliferación de marcas, saturación publicitaria, uso de tecnologías de la información y comunicación, crisis económica, etc.

Bajo este contexto, se pretende, en este artículo, despejar dudas conceptuales en torno a los tres elementos de interés (identidad, imagen y reputación), para posteriormente reconocer las maneras en que las capacidades materiales e inmateriales del ser humano, cerebro y mente, responden a estímulos externos de comunicación y publicidad, llevándolo a tener la capacidad de aceptar, rechazar o ser indiferente porque finalmente, será el individuo quien hace uso "de la libertad de un ser racional" (Kant, 2006: 631).

\section{Identidad - Imagen - Reputación. Elementos constitutivos de la marca}

Tanto en el ambiente académico como en el empresarial persisten problemas para identificar las diferencias entre imagen, identidad y reputación. Esta ambivalencia ha generado un sinnúmero de problemas de orden estratégico en el planteamiento de comunicaciones empresariales, además de los inconvenientes que ello implica al momento de realizar investigaciones sobre las percepciones que los consumidores tienen de una marca u organización. 
La diferencia más significativa se da en la generación de los conceptos; en este caso se puede sostener que la identidad se construye dentro de la empresa como parte integral de su gestión de marca. Por el contrario, la imagen es el resultado de acciones corporativas que redundan en las percepciones que los públicos tienen de la marca. Aaker aclara que la identidad es una parte primordial del desarrollo empresarial, pues representa "un conjunto único de asociaciones que el estratega aspira a crear o mantener. Estas asociaciones representan la razón de ser de la marca implicando una promesa de los integrantes de la organización a los clientes” (Aaker, 1996: 71). En contraposición, Toro es partidario de ubicar la identidad en el lado de la percepción, es decir, en el espacio de los stakeholders: "Podemos definir la identidad de una marca como aquel conjunto de asociaciones que percibe el consumidor a través de cualquier tipo de contacto con la marca y que transmiten sus atributos y beneficios" (Toro, 2009: 53). Estas dicotomías conceptuales, en las que un autor considera la identidad en la margen de la empresa (Aaker), y el otro la ubica en la margen de los stakeholders (Toro), es lo que ha provocado una importante disparidad en el tratamiento académico de este tema.

Será de vital importancia, tanto para académicos como para estrategas de la marca, no mezclar los espacios que estos dos conceptos irradian, pues allí radica un grave problema en la estrategia publicitaria y del diseño: la falta de un método riguroso para conceptualizar y ubicar el sentido de las teorías, que se tornan "populares" en el manejo práctico y en el ejercicio de la actividad diaria, pero dicotómicas en el sentido útil de la materialización conceptual. Un ejemplo evidente en este aspecto representa la forma en que algunas grandes y pequeñas empresas continúan denominando a los manuales de identidad corporativa como "manual de imagen corporativa". En este sentido, han sido los propios profesionales de la publicidad, la comunicación y el diseño los causantes de esta mala utilización, que no se ciñen a la realidad conceptual y terminológica.

Sin embargo, la psicología cognitiva y la neurociencia entran a jugar un papel clave, tratando de ayudar a esclarecer conceptos, como el de imagen, que hace referencia a las asociaciones que percibe el consumidor. Cuando un estímulo externo es captado por medio de los sentidos, dicho estímulo puede evocar una marca. Este fenómeno se presenta porque la mente del consumidor construye lo que se denomina redes de asociaciones.

Las redes de asociaciones son mapas mentales donde se tejen todos aquellos conceptos que relacionamos con la marca, que pueden ser atributos tangibles, percepciones o valores. Estas redes se construyen por asociaciones de conceptos que el cerebro ha almacenado previamente por medio del aprendizaje, el pensamiento y la memoria. Las representaciones mentales que construye el individuo surgen por percepciones y vivencias personales, lo que los convierte en subjetivas; las vivencias de un individuo son diferentes a las de cualquier otra persona, de esta manera las percepciones se convierten en una elaboración individual y personalizada de la realidad (Braidot, 2005). Así, aunque las experiencias sean subjetivas, son verdaderas y "no podemos dudar de las experiencias propias" (Solomon, 2009: 70). Los valores de la marca 
pueden ser jerarquizados según su grado de importancia o por afinidad con los intereses de los stakeholders, ya sean estos intereses individuales o colectivos.

Los aportes que disciplinas como la psicología cognitiva, la filosofía y la neurociencia pueden hacer en el campo de la identidad y la imagen, radican en el hecho de comprender que en el ser humano las redes de asociaciones mentales pueden ser modificadas porque "los modelos de relaciones entre ideas, así como las ideas mismas, son susceptibles de ajuste bajo la presión de los hechos. Por supuesto, estos hechos son producto de la aplicación de ideas previas a nuestras sensaciones" (Harré, 2002: 253). Si logramos entender cómo modificar estas estructuras neuronales, es posible que se pueda aplicar a la estructura del pensamiento de los diferentes stakeholders y, en especial, la del consumidor final; esta posibilidad la describe Ratey cuando relata que "Gracias a su asombrosa plasticidad, el cerebro humano modifica sin cesar sus conexiones y aprende, no sólo por medio del estudio académico, sino de la experiencia” (Ratey, 2002: 66).

Gracias a esa plasticidad cerebral los stakeholders piensan y sienten de manera diferente, porque cada individuo "tiene su propio entendimiento agente y pasivo. Sólo así puede cada hombre llamarse racional, y sólo así se explicaría que los hombres, al mismo tiempo, piensen cosas contrarias" (Fischl, 2002: 195). O como lo precisa Guzmán: "nuestras formas normales de actuar determinan nuestra propia estructura mental, mucho más individualizada y distinta de lo que puede serlo nuestro rostro físico o nuestras huellas dactilares" (Guzmán, 2001: 39). Por su parte, Hax y Majluf afirman que "podemos decir qué es la estrategia, pero no podemos proponer una fórmula universal aplicable a cualquier empresa concebible que enfrenta cualquier tipo de medio que tenga una validez general" (Hax y Majluf, 1997: 23).

Dadas estas evidencias y las otorgadas por importantes investigadores de la identidad de marca (Ind, 1992; Kapferer, 1992; Sanz de la Tajada, 1996; Van Riel, 1997; Chaves, 2008; Keller, 2008; Capriotti, 2009; Ávalos, 2010), se entiende que la identidad se construye en los cimientos de la empresa, al considerar aspectos vitales como el contexto de la marca, los valores empresariales, su sostenibilidad en el tiempo, su diseño corporativo (colores, logotipos, embalajes, etc.), su comunicación (publicidad, patrocinios, eventos...), entre otros. Por consiguiente, la identidad será lo que la empresa conciba a partir de su estrategia, una proyección de la realidad, a través de todas sus manifestaciones (García, 2011).

La forma en que la empresa concibe la identidad será clave para que la marca sea aceptada y reconocida por los diferentes públicos. A tal efecto, Gázquez y Sánchez, consideran que la marca debe reunir tres propiedades básicas para que su identidad sea aceptada:

"Una marca tendrá credibilidad cuando el mundo que propone pueda ser asociado con el producto o servicio al que la marca confiere valor y significado. Por otro lado, la legitimidad está más vinculada a las acciones de la empresa que a las expectativas de los consumidores. Si la coherencia contribuye a lograr la credibilidad, lo que le confiere legitimidad a una marca es la continuidad a lo largo del tiempo. La tercera propiedad para que la identidad de una marca sea aceptada por parte del público objetivo es la afectividad, referida a los 
contratos psicológicos implícitos entre los consumidores y sus marcas preferidas. Así, la marca incrementará sus posibilidades de ser elegida y preferida si consigue estimular las emociones del público" (Gázquez y Sánchez, 2004: 65).

Así, la compañía podrá crear una relación duradera con sus públicos a través de una identidad que proyecte una fuerte personalidad de marca, que interprete la cultura corporativa, que respalde la calidad del producto o servicio, para posteriormente convertirse en el mejor resultado de la estrategia de identidad: una sólida y única imagen de marca.

Al contrario de lo que sucede con la identidad, la imagen se crea en el imaginario de los stakeholders, en su memoria, como un intangible de recepción. Será un proceso acumulativo, de evaluación y asociación que se desarrolla en la mente de los públicos, permitiendo de esta manera configurar la estructura cognitiva del sujeto (Ostberg, 2007). Se puede entonces señalar que la imagen es subjetiva, relativa a las propias percepciones del individuo. Dichas percepciones subjetivas serán el resultado de todos los inputs que recibe el sujeto durante su "experiencia" personal con la marca: relación directa con sus productos o servicios, imagen creada a partir de lo que percibe por la publicidad, o incluso comentarios de un amigo o familiar. Al respecto, Kapferer plantea que "la imagen se refiere a la forma en que ese público interpreta el conjunto de señales procedentes de los productos, servicios y comunicaciones emitidas por la marca” (Kapferer, 1992: 44).

La imagen es entonces la base sobre la que se soportan las percepciones que tienen todos los stakeholders sobre la empresa. Esta concepción es compartida por investigadores de la identidad e imagen de marca (Ávalos, 2010; Brujó, 2010; Hatch y Schultz, 2010; García, 2011); así, la imagen de marca se convierte en una red de memoria asociativa basada en las percepciones de los consumidores (Keller, 2008), o también es interpretada como la fuerza que incita nuevas preferencias, decisiones, emociones y convicciones hacia la marca (Costa, 2004).

Dichas percepciones provienen principalmente de dos fuentes: la propia identidad que genera la marca y sobre todo de los ruidos o señales externas que reciben los stakeholders (Cerviño, 2002). Lo que equivale a decir que la imagen de marca que se crea en la mente de los usuarios no siempre está generada por los mensajes emitidos por la empresa, pues también puede presentarse que lo que los usuarios y no usuarios de una marca piensen sobre ella provenga de gran cantidad de emisores, como por ejemplo las opiniones de otros clientes, lo que se emite en las redes sociales a través de internet, o lo que emiten los medios de comunicación a partir del free press. Finalmente, a las percepciones de los consumidores se deben anexar las asociaciones y las expectativas que la imagen de marca genera en la mente:

"It bears repeating that a brand is created, not only as a result of a marketer's activities (the stimulus or 'input') but also, critically, as a result of the consumer's reading of and reaction to those activities (the 'take out'). From the marketer's perspective, a brand is a promise, a covenant. From the consumer's, it is the set of associations, perceptions and expectations existing in his or her mind. Brand associations are created, sustained and enhanced by every experience and encounter a consumer has with the brand" (Batey, 2008: 4) 
En resumen, se puede entender que la identidad se crea al interior de la organización como parte de su estrategia empresarial -concepto de emisión-, mientras que la imagen se construye y permanece en la mente de los stakeholders-concepto de recepción-.

Pero son las sutiles diferencias entre los conceptos de imagen y reputación los que más confusión generan. Para Villafañe la diferencia sustancial entre uno y otro provienen del umbral de cada constructo, pues considera que "mientras que la reputación corporativa es la expresión de la identidad de la organización y del reconocimiento de su comportamiento corporativo, la imagen proyecta su personalidad corporativa y es, en este sentido, el resultado de la comunicación en todas sus formas" (Villafañe, 2004: 29). Es decir, la reputación sería el resultado de la identidad corporativa, mientras que la imagen, el resultado de lo que las comunicaciones de la empresa proyecten en los stakeholders.

Si bien es cierto que la reputación tiene un sentido más estructural que la propia imagen, quizá no se podría asegurar que la reputación sea necesariamente producto de la identidad; también lo puede ser del conocimiento, fundado o no, de los stakeholders. En términos concretos, podemos acudir a lo que Olcese, Rodríguez y Alfaro (2008) concretan como diferencias entre uno y otro concepto.

Tabla I. Diferencias entre imagen y reputación corporativas

\begin{tabular}{|l|l|}
\hline Imagen corporativa & Reputación corporativa \\
\hline Se basa en percepciones & Se basa en resultados \\
\hline Es difícil de objetivar & Es verificable empíricamente \\
\hline Genera expectativas asociadas a la oferta & Genera valor, consecuencia de la respuesta \\
\hline Carácter coyuntural y efectos efímeros & Carácter estructural y efectos duraderos \\
\hline En parte es resultado de la comunicación & Resultado del comportamiento corporativo \\
\hline Se construye fuera de la empresa & Se genera en el interior de la empresa \\
\hline
\end{tabular}

Fuente: (Olcese, Rodríguez y Alfaro, 2008: 178).

Se entiende que tanto la imagen como la reputación se sitúan como condición cognitiva del sujeto, pero las formas en que dichas asociaciones se realicen serán las que generen las diferencias más significativas. En definitiva, se puede considerar una diferencia importante entre ambos conceptos: la imagen forma parte de las percepciones que los stakeholders tienen de la marca; la reputación la ultrapasa y determina un conocimiento más profundo de la marca, pero sin dejar de ser, al igual que la imagen, una percepción subjetiva. 


\section{Reputación, esencia de una imagen fundamentada}

Aunque se ha descrito la diferencia entre conceptos tan próximos como reputación, imagen e identidad, es necesario definir qué representa la reputación corporativa como punto de partida que permitirá interpretar la manera en que los stakeholders perciben la información de las marcas, para posteriormente convertir en sus mentes dicha información en un intangible básico para el éxito empresarial.

La reputación corporativa es considerada una teoría en plena construcción, sin bases teóricas fuertes, ya que incluso, aún no existe unidad de criterio sobre los elementos que constituyen el concepto (Suviri, 2010); además, en absoluto se ha producido un consenso sobre su definición (López y Iglesias, 2010). La base de esta situación puede originarse en tres condiciones esenciales: el hecho de haber sido abordado por distintas disciplinas, su carácter multidimensional y su alto grado de intangibilidad (Martínez y Olmedo, 2009). Ante la falta de consenso, se puede valorar la forma en que Villafañe entiende este concepto:

“La expresión de la armonía entre la lógica central de la empresa, que enfatiza los resultados económicos y financieros y la fortaleza de su oferta comercial, y las lógicas marginales, orientadas hacia aquellas políticas y comportamientos corporativos como la gestión del capital humano, sostenibilidad de sus acciones, o la ya citada responsabilidad social corporativa" (Villafañe, 2004: 20).

En esta definición se descubre que la reputación es un nivel más avanzado en el conocimiento que los stakeholders tengan de la empresa o de una marca determinada. En tal sentido, Costa (2009) cree que la reputación es el reconocimiento valorativo que los públicos interesados otorgan a la empresa, lo que implica que los stakeholders deben tener una mayor y mejor información sobre la organización a la que están juzgando.

Los parámetros axiológicos o juicios de valor que los stakeholders tienen de las empresas son uno de los elementos que aún no se vislumbra hasta qué punto son evidentes en la mente de las diversas partes interesadas. Siguiendo con Villafañe (2005), este autor indica que el reconocimiento de dichos valores se presenta en cuatro ámbitos muy bien diferenciados: económico, comercial, laboral y social. Si entendemos que la reputación representa la percepción que tienen los diversos stakeholders de una empresa, aspectos como sus acciones laborales o sociales requieren de un mayor conocimiento. Este no se presenta como efecto de la publicidad, o de las concepciones que puede tener una persona de un producto simplemente observando un envoltorio en el supermercado o al escuchar un comentario de un amigo. Dadas estas circunstancias, es pertinente acudir a la definición que presentan Rey y Bartoli en la que asumen que "la reputación no es la imagen corporativa de una organización. La reputación es un juicio o valoración que se efectúa sobre dicha imagen en la mente de los públicos" (Rey y Bartoli, 2008: 94). Para corroborar aún más este enfoque del conocimiento de los stakeholders, es pertinente rescatar la idea que Quevedo tiene sobre la creación de la reputación: 
"La reputación es un activo de construcción social, que es creada y mantenida a través de un proceso de legitimación, e implica una continua sucesión de evaluaciones puntuales de las actuaciones de la empresa en cada momento, cuyo veredicto debe basarse en criterios de legitimidad. La acumulación de valoraciones homogéneas en períodos sucesivos redundará en la reputación de la empresa" (Quevedo, 2003: 2).

Una consideración importante de mención es que la reputación no es el resultado de una comunicación efímera por parte de la empresa, se construye a través de un largo proceso en el que los stakeholders se involucran de manera continua con la marca.

Uno de los problemas más importantes con los que los directores de comunicación deben enfrentarse es que el camino a la creación de reputación es de largo aliento e implica altos costes, porque incluso cualquier tipo de reputación que haya construido la organización puede cambiar abruptamente, como lo definen Lange, Lee y Dai (2011) al introducir nueva información de comportamientos no deseados, lo que inmediatamente generará un cambio en las relaciones entre la empresa y sus stakeholders. De esta manera, lo que Capriotti (2009) denomina "consumo de información" que realizan los individuos se materializará a partir de la comunicación de la empresa. Es allí donde la comunicación entra a jugar un papel primordial en la construcción de la reputación.

Desde la perspectiva de los conceptos aglutinadores, algunos teóricos (Fombrun, 1996; Dowling, 2001; Argenti y Forman, 2002; Quevedo, 2003; Villafañe, 2004; Alsop, 2004; Hannington, 2006; Capriotti, 2009, Earl y Waddington, 2012), establecen elementos comunes que integran el concepto de reputación. Así, para Capriotti la reputación es "la estructura o esquema mental que una persona, grupo de personas o entidades tiene sobre un sujeto (organización, producto, servicio, país, etc.), compuesta por un conjunto de asociaciones que dichos públicos utilizan para identificar, diferenciar y evaluar a dicho sujeto de otros" (Capriotti, 2009: 102). Mientras que para Quevedo la reputación es "la percepción, a lo largo del tiempo, que la empresa mantiene una actitud legítima con todos y cada uno de sus participantes, tanto en términos de comportamiento como informativos" (Quevedo, 2003: 23). En estas dos definiciones se mantiene la constante que relaciona la reputación con las percepciones de los diversos stakeholders a lo largo de un tiempo determinado, es decir, no se trata de una “imagen de marca" que sería el nivel perceptual primario, sino que trasciende a un conocimiento mucho más profundo del objeto de análisis, donde se pueden incluir aspectos tan específicos como la oferta comercial, las políticas y comportamientos corporativos, los resultados económicos y financieros, e incluso hasta un conocimiento de las acciones en materia de Responsabilidad Social Corporativa.

Aunque no exista consenso en la definición ni en los elementos sustanciales de la reputación corporativa, sí se pueden relacionar algunos elementos relevantes que le dan trascendencia al concepto. Bajo estas reflexiones la reputación corporativa se puede considerar como un intangible que las empresas gestionan como consecuencia de sus resultados económicos y de su comportamiento socialmente responsable (López y Sebastián, 2009), o incluso como la respuesta de los individuos a la posición de la empresa ante una crisis (Fernández, 2011). 
Al contrario de lo que ocurre con la conceptualización de la reputación corporativa que no ha logrado una línea unificadora, la gestión de la reputación a nivel empresarial ha descubierto parámetros y circunstancias que le permiten un importante crecimiento estratégico. Una parte fundamental para que se genere este avance significativo tiene correspondencia con la variedad de investigaciones que en los últimos años se han realizado sobre los efectos en la reputación de las acciones empresariales. Se pueden encontrar estudios tan diversos como los efectos que la eficiencia en el trabajo y la productividad laboral generan en la reputación (Stuebs y Sun, 2010); la percepción de los públicos sobre la sinceridad empresarial y su influencia en la reputación (Hyo-Sook, 2011), la influencia que tienen los informes de Responsabilidad Social Corporativa (Nikolaeva y Bicho, 2011), o incluso la incidencia de los valores de confianza empresarial en la creación de reputación (Komisarjevsky, 2012).

Uno de los valores más importantes en la gestión de la reputación corporativa corresponde a la comunicación que la empresa realice con sus stakeholders. Así lo interpreta Hannington cuando asegura que "no es sólo cómo usted comunica, sino también qué comunica lo que importa. Su plataforma de comunicaciones es uno de los elementos críticos sobre los que se construye la reputación" (Hannington, 2006: 85). Dada la importancia de lo que la empresa comunique, la gestión comercial no será la única herramienta de diálogo con los grupos de interés, pues aunque esta comunicación masiva permite abarcar más stakeholders, también se deberá poner en práctica una información relacional, es decir, una comunicación “directa, selectiva, personal o personalizada. Continuada e informacional. Y, sobre todo, ética” (Costa, 2009: 111).

La reputación solo se logra cuando la empresa alcanza altos estándares de credibilidad y de confianza, lo que permitirá una relación mucho más cercana y estrecha entre la empresa y sus stakeholders (Orozco y Roca, 2011). Si la empresa tiene una excelente reputación tendrá la oportunidad de reforzar los mensajes de calidad de los productos y cargar primas de precios superiores (Salinas, 2007), pero incluso hasta la marca que tenga la mejor reputación puede sufrir cambios repentinos cuando aparecen escándalos públicos, así lo corroboran Lange, Lee y Dai al mencionar que "reputation is rooted in the organization's historical behavior and associations but can be abruptly changed if new information about the organization's past behavior comes to light" (Lange, Lee y Dai, 2011: 154). Estos escenarios de crisis de reputación son las peores circunstancias que puede sufrir una empresa, dado que las percepciones de los stakeholders pueden cambiar y tornarse desfavorables, incluso, luego de largo tiempo de haber disfrutado de una excelente relación con sus distintos grupos de interés.

Las crisis que han tenido que afrontar un número importante de empresas en los últimos años han alumbrado un nuevo paradigma en la reputación corporativa: el impacto que los stakeholders han generado en el descrédito de dichas compañías. La globalización y las Tecnologías de la Información y Comunicación (TIC) le han otorgado un importante poder a los grupos de interés, sobre todo porque nuestras conexiones y nuestra interdependencia son globales, "lo que ocurre en un lugar influye en las vidas y las oportunidades vitales de personas de todos los lugares” (Bauman, 2010: 44). Sin em- 
bargo, y muy a pesar de la presión que ejercen los stakeholders en las decisiones empresariales, aún hoy se continúan sucediendo escándalos que han menoscabado la reputación corporativa de varias compañías. No obstante, el hecho de que los stakeholders tengan cada vez más poder no representa un problema para la comunicación ni para la actuación de las empresas, es más bien una oportunidad para que se materialice una comunicación transparente, convertida en diálogo, en donde la confianza y la credibilidad sean el soporte para una relación de mutuo beneficio.

Antes de pasar al análisis de la forma en que piensan los consumidores y, tomar como referencia sus aspectos mentales, como base para la interpretación de la reputación corporativa, se debe reconocer que este proceso posee una importante variedad de matices, gracias a las diferencias entre marcas, medios de información, pero, sobre todo, a la nueva concepción que se tiene de la noción stakeholder.

El concepto stakeholder significa aquel que es depositario de una apuesta por parte de la organización, sin los cuales la misma no tendría sentido dado que "the stakeholder was originally defined as those groups without whose support the organization would cease to exist” (Freeman, Harrison, Wicks, Parmar y Colle, 2010: 30). Así, los diferentes actores que tienen relación directa o indirecta con la empresa comienzan a tener un valor preponderante, ya que serán considerados parte fundamental de su desarrollo. A raíz de este cambio en la concepción de los clientes o públicos, se relacionan con la empresa gran variedad de stakeholders que en la literatura académica pueden aparecer con dos significados: grupos de interés y/o partes interesadas.

Este nuevo paradigma en la concepción de los grupos de interés permite que se genere una ampliación de las responsabilidades de la empresa y, corrobora la idea de que una misma empresa podrá tener diferentes tipos de reputación, según el grupo de interés sobre el que se evalúen sus acciones. La compañía ya no solo centra sus acciones estratégicas exclusivamente en unos grupos específicos, sino que comenzará a fijarse en distintos grupos que posean diferentes intereses, reclamaciones o exigencias. Además, ante la diversidad de stakeholders (consumidores, proveedores, empleados, accionistas, competidores, entre otros), se debe ser consciente de que la reputación impactará a muy diversos tipos de stakeholders, incluso desde aspectos tan diversos como la calidad de los productos, la Responsabilidad Social Corporativa, el servicio o la ética y transparencia empresarial (Doorley y García, 2011).

Dado que los stakeholders no responden a los mismos estímulos, las organizaciones deberán investigar y comprender la manera en que se construye la reputación en sus públicos. De hecho, Dowling (2001) asume que hay cuatro tipos de stakeholders evaluados bajo la perspectiva de la reputación: grupos normativos, funcionales, de difusión y finalmente los consumidores. En este punto de la relación entre las marcas y los consumidores es donde entran a jugar un papel decisivo teorías como el branding emocional, que le otorga una importancia mayor a las experiencias sensoriales (Gobé, 2005), y la psicología cognitiva que intenta explicar cómo las percepciones influyen en la mente de los stakeholders. 


\section{4. ¿Cómo piensan los stakeholders?}

Los conceptos de imagen y reputación tienen un punto de encuentro en común: el cerebro. Camargo (2009) se refiere a él como la "caixa preta", la caja negra de la ciencia moderna. El cerebro es el órgano del cuerpo humano encargado de procesar la información que se capta a través de los sentidos y es el responsable de los procesos cognitivos. En él se pueden dar dos tipos de procesos u operaciones de mutua dependencia: las fisiológicas (procesos cerebrales) y las cognitivas (operaciones mentales). Estos procesos y operaciones de carácter material e inmaterial no son contradictorios sino complementarios, este concepto fue expuesto inicialmente por Tomás de Aquino cuando se refirió a las potencias del alma como orgánicas (aquellas que necesitan de un órgano corporal) o inorgánicas (las que no necesitan de un órgano corporal como el entendimiento y la voluntad), es así entonces "como también el pensamiento presupone las representaciones sensibles y él es a su vez presupuesto para el querer, síguese que, indirectamente toda vida espiritual depende de la sensible" (Fischl, 2002: 194). Algunos procesos fisiológicos tienen relación con el control, la coordinación del movimiento y el comportamiento. Por otra parte, las operaciones mentales son las que llevan a cabo el procesamiento de las estrategias para pensar, las cuales se transforman en habilidades por medio de la práctica y posteriormente se convierten en la facultad que tienen los individuos para aplicar el proceso denominado pensamiento. Pensamiento y mente son dos conceptos que interactúan en el interior de cada consumidor y donde las marcas luchan por estar en uno o en ambos espacios.

Pensar es categorizar, deducir, verificar hipótesis, resolver problemas, tomar decisiones, inventar; estas son características que permiten organizar el pensamiento. En el diccionario general de sinónimos y antónimos, se encuentra que:

"Pensar es simplemente poner en uso las facultades mentales; considerar es pensar con detenimiento; reflexionar es examinar atentamente todas las ideas cuyo conjunto interesa o llama la atención; meditar es emplear en este examen el uso de la imaginación. Para pensar se necesita objeto; para considerar, interés; para reflexionar, crítica; para meditar, imágenes" (Blecua, 1999: 892).

El pensamiento, como proceso cognitivo superior de la mente, implica una actividad integral de operaciones cognitivas básicas donde intervienen la percepción, la memoria, la atención, el análisis, la comprensión, el aprendizaje y las representaciones mentales. La mente, del latín mens, mentis "potencia intelectual del alma", es el conjunto de actividades conscientes y no conscientes "que se producen por la interacción y comunicación entre grupos de neuronas que dan forma a los pensamientos y sentimientos" (Braidot, 2005: 16). Es la facultad del cerebro responsable del entendimiento donde se realizan algunos procesos cognitivos. Entre los más destacados se encuentran: el pensamiento, la atención, la memoria y la percepción. Esta última considerada una variable importante para entender la relación marca-percepciónconocimiento-consumidor.

Todo el proceso del conocimiento se inicia cuando los cinco sentidos (vista, olfato, gusto, oído, tacto) reciben estímulos externos al individuo, estos estímulos se captan en forma de sensaciones, las cuales a su vez se transforman en impulsos 
nerviosos que van al cerebro por medio del sistema nervioso y las conexiones sinápticas de las neuronas, para luego ser interpretadas mediante procesos cognitivos (mentales) y posteriormente quedar guardados en la memoria. Todo esto se logra mediante el proceso de la percepción. Desde una perspectiva filosófica, Locke considera que la "percepción es la primera facultad de la mente que se ejerce sobre nuestras ideas, de forma que es la primera y más simple de las ideas que recibimos por medio de la reflexión; algunos la llaman con el nombre de pensar" (Locke, 1970: 69). La percepción es innata al ser humano y está presente en todo el desarrollo del individuo. Este principio que es estudiado por la psicología cognitiva fue planteado desde la filosofía por Locke en 1690 refiriéndose a que "nuestros sentidos se ocupan con objetos particulares sensibles y conducen a la mente percepciones distintas de las cosas, de acuerdo con los diversos modos con que estos objetos les afectan" (Locke, 1970: 48). La percepción permite obtener información del exterior de modo significativo para captar la realidad en forma organizada, "la realidad es lo que el cliente percibe que es" (Braidot, 2005: 47). La percepción es un término polisémico que puede ser estudiado desde varios puntos de vista disciplinares:

- Filosófico: La percepción no es solo un reflejo de la realidad, es parte de la ella y como tal debe ser estudiada.

- Físico: Ondas electromagnéticas que influyen en nuestros sentidos.

- Psicológico: Los procesos fisiológicos y las operaciones mentales que influyen en la percepción.

Para los efectos de este artículo concentraremos nuestro análisis en el ámbito psicológico. Aunque muchas aportaciones importantes vienen desde la filosofía, también se constituyen en una fuente importante para la comprensión del concepto. Una aproximación a este respecto es la que hace Hume en su obra Tratado de la naturaleza humana, publicado en 1739, y en donde considera que todas las percepciones de la mente humana se concretan en dos clases distintas: las impresiones y las ideas (Hume, 1984). La diferencia entre ambas está en el nivel de impacto que tenga en los sentidos, siendo así que si la percepción es fuerte, esta se convierte en sensación, emociones o sentimientos, para luego dar paso a la impresión. Las sensaciones son impresiones que el individuo recibe del entorno y desencadenan una serie de conexiones que pone en acción un mapa neuronal determinado. Son de carácter físico, por ejemplo, la sensación de calor, frío, duro, blando, agudo, grave, etc. La emoción es la agitación, perturbación o conmoción de la mente que se manifiesta de forma física, por ejemplo, alegría, tristeza, sorpresa, miedo, desprecio, etc. Los sentimientos son estados de ánimo, por ejemplo, nostalgia, satisfacción, decepción, etc. Las ideas, por su parte, son impresiones más débiles, son las imágenes que se presentan cuando el individuo piensa y razona pero que no causan un impacto duradero en la mente del individuo. Esas imágenes débiles son lo que posiblemente asociamos con el concepto de “imagen de marca”. Entonces surge la pregunta: ¿Es apropiado hablar de imagen de marca, impresión de marca o ambas?

Ya sea que el concepto se aborde desde la filosofía o desde la psicología cognitiva, los consumidores reciben estímulos de las marcas, las cuales llegan a ser tan fuertes desde aspectos como: empaque, nombre, mensajes publicitarios, etc., que 
pueden generar impresiones intensas, hasta convertir un sentimiento positivo hacia una marca en un vínculo emocional duradero, incluso puede llegar a "grabarse" de forma permanente en la mente. Pero también puede ocurrir lo contrario, que algún aspecto de la marca genere un impacto negativo fuerte, generando un sentimiento de rechazo duradero. Pero si las percepciones que recibe el consumidor son débiles o imperceptibles, la imagen que se creará no será fuerte y posiblemente termine convirtiéndose en una idea.

La percepción es un proceso tan complejo que debe desarrollarse paralelamente en tres sentidos: desde la forma como el mundo influye en el consumidor (de afuera hacia adentro), como el consumidor influye en el entorno (de adentro hacia fuera) y los aspectos internos del consumidor que también influyen en la percepción (de adentro hacia adentro). Lo anterior hace que las percepciones sean captadas con atención e influyen en ellas muchos aspectos internos del individuo, lo que le da a la percepción un carácter parcial o subjetivo. Esta subjetivación se presenta cuando al momento de interpretar la información recibida por los sentidos, el individuo parte de una predisposición mental (personal, social, educativa, cultural) que afecta a lo que se percibe. En otras palabras, el consumidor percibe lo que desea percibir.

Tabla II. Campos de desarrollo de la percepción

\begin{tabular}{|l|l|}
\hline $\begin{array}{l}\text { De afuera hacia } \\
\text { adentro }\end{array}$ & $\begin{array}{l}\text { Cuando el consumidor ve, toca, huele, escucha o degusta los elementos constitutivos de una } \\
\text { marca, la percepción de este cambia modificando su estructura mental. Esa modificación puede } \\
\text { ser positiva, negativa o indiferente que aunque no presenta cambios significativos los estímulos } \\
\text { pasaron los filtros de los sentidos. Es el caso de la reputación, que desea construir una empresa, } \\
\text { puede generar una impresión fuerte o una imagen débil en la mente de los consumidores. }\end{array}$ \\
\hline $\begin{array}{l}\text { De adentro hacia } \\
\text { fuera }\end{array}$ & $\begin{array}{l}\text { Se presenta cuando lo imaginado parte de las experiencias previas del individuo (educación, cul- } \\
\text { tura, personalidad, etc.) e influye para que la marca modifique una o varias de sus características } \\
\text { constitutivas. Es decir, la marca es afectada por el consumidor. Es cuando la marca debe realizar } \\
\text { cambios porque los stakeholders lo exigen. }\end{array}$ \\
\hline $\begin{array}{l}\text { De adentro hacia } \\
\text { adentro }\end{array}$ & $\begin{array}{l}\text { Surge cuando las experiencias previas (educación, cultura, personalidad, etc.) afectan al mismo } \\
\text { tiempo las percepciones internas. Cuando lo imaginado modifica la estructura mental del sujeto. } \\
\text { Cuando un consumidor o grupo de consumidores tiende a ver todo lo que sucede a su alrededor de } \\
\text { forma optimista, es posible que cualquier mensaje de una marca aunque en apariencia sea nega- } \\
\text { tivo, seguirá siendo visto por el consumidor de forma optimista. }\end{array}$ \\
\hline
\end{tabular}

Fuente: Elaboración propia. 
La percepción en la mente de los consumidores no es estática, por el contrario, es activa y dinámica, se actualiza en la medida que avanzan los desarrollos tecnológicos, se incorporan nuevos hábitos culturales y los cambios de los mercados a ritmo acelerado. A este respecto Mora considera que percibir es un proceso de aprendizaje a lo largo del tiempo "que es lo mismo que decir un constante modelado bioquímico, anatómico y fisiológico de nuestro cerebro" (Mora, 2009: 44). De la forma como un niño aprende a entender los estímulos que sus sentidos captan (percepción), estará condicionando todas las actividades perceptivas en el futuro (Landan, 1987; Piaget, 1994). Este punto es importante para comprender por qué las marcas intentan persuadir por medio de la percepción sensorial y cómo los consumidores interpretan las marcas de manera diferente, aunque todos los stakeholders se encuentren expuestos a los mismos estímulos de marketing, publicidad, relaciones públicas, comunicación, etc.

\section{Conexión imagen-reputación-consumidor}

Existen dos posiciones frente al estudio de los procesos mentales. En primer lugar, se encuentran investigadores como (Martínez-Freire, 1995; Pinker, 2001; Ruesch y Bateson, 2008), que asocian las operaciones mentales con programas de ordenador y se conoce como Modelo del ordenador o enfoque de Inteligencia Artificial. Esta teoría defiende que la mente es como un ordenador que procesa información por medio de estímulos (inputs) y ofrece respuestas (outputs). Por otra parte, las teorías de (Sternberg, 1986; Ratey, 2002; Llinás, 2003) afirman que los procesos mentales se dan en forma de conexiones similares a las que realizan las neuronas, este modelo también es conocido como Modelo del cerebro o hipótesis conexionista o asociacionista. Esta teoría asociacionista considera que la percepción es como un mosaico de sensaciones, donde se perciben sensaciones aisladas, las cuales en su conjunto forman la percepción general del objeto, es decir, el conjunto de elementos que constituyen una marca forman la percepción general de ella, o que se conoce como la imagen de marca. Lo anterior se puede comprender mejor cuando se asume al individuo como un ser humano constituido por cuerpo y alma, donde el alma "es espíritu de vida, espíritu intelectual, espíritu racional siempre en movimiento" (Saranyana, 1989: 103).

Otro grupo de teóricos, los de teoría de la Gestalt, argumentan diferencia de lo propuesto por los conexionistas, que los estímulos no se perciben de forma aislada sino que lo hacen de una vez en conjunto y de forma organizada. Sin embargo, el concepto de imagen de marca puede tener una interpretación clarificadora si se toma como referencia los aportes realizados por Hume (1984), desde la filosofía, cuando consideraba que las impresiones y las ideas pueden ser de carácter simple o complejo. Las percepciones simples son aquellas que son indivisibles en sí mismas, por ejemplo el concepto de color, forma, textura. Por otro lado, las percepciones complejas están compuestas por la suma de percepciones simples que conforman el objeto y sí admiten separación porque son diferentes entre sí.

- Percepciones simples: Color amarillo. Textura lisa. Forma circular. Sabor agridulce. 
- Percepciones complejas: Naranja = color + textura + forma + sabor. Las percepciones simples se pueden distinguir unas de otras.

Estas posturas tienen su origen en la propuesta de Hume (1984), quien planteó que los individuos pueden percibir el entorno por las características individuales que van sumando hasta construir la imagen general (conexionistas), o la posibilidad de percibir todas las características al mismo tiempo (teoría de la Gestalt). Esta postura es interesante como punto de partida de análisis de futuras estrategias de comunicación, porque para la empresa X el empaque de su producto puede ser percibido por la mente de los consumidores como una impresión (percepción fuerte) y una percepción compleja (un elemento más de la imagen). Pero para la empresa Yel empaque puede ser percibido como una idea (percepción débil) y una percepción simple (es el valor más importante de la imagen). Ahora bien, en el caso de la reputación estaríamos hablando de una impresión (percepción fuerte) acompañada de una percepción simple (es único e indivisible). Podemos afirmar entonces que el consumidor puede percibir, estructurar y construir en su mente muchas imágenes de marca, pero solo la reputación puede lograr que las percepciones se perciban y se fijen en la mente de forma permanente. En este caso, estaríamos hablando de una reputación construida por impresiones de marca, es decir, fuertes percepciones que el consumidor ha ido construyendo, de forma coherente con el paso del tiempo, hasta llegar a convertir las sensaciones, las emociones y los sentimientos desde y hacia la marca en vínculos duraderos y/o permanentes.

Ahora bien, posiblemente uno de los autores que ha logrado integrar el funcionamiento de la mente y del cerebro humano ha sido Gardner (2008), psicólogo con experiencia en psicología cognitiva y neurociencia quien escribió a finales de la primera década del siglo XXI el libro titulado: Las cinco mentes del futuro. En él describe cinco tipos de mente, cómo funcionan y cómo pueden desarrollarse. Estos son: la mente disciplinada, la mente sintética, la mente creativa, la mente respetuosa y la mente ética. El análisis de esta clasificación ha sido fundamental para comprender que los seres humanos piensan diferente, porque Gardner las considera "como usos generales de la mente que se fomentan en la escuela, en el mundo de las profesiones liberales y en el lugar de trabajo” (Gardner, 2008: 17). Lo anterior abre la posibilidad de conocer y comprender qué pasa en la mente de los stakeholders cuando están expuestos a algún elemento constitutivo de la marca (logotipo, empaque, etiqueta, exhibición, publicidad, promoción, etc.). A este respecto Zaltman (2004), miembro de la Mind, Brain and Behavior Initiative, de la Universidad de Harvard, abre el debate que hemos venido desarrollando en este artículo, definiendo que existen dos tipos de mentes, la mente de los directivos de empresa y la mente de los consumidores. Esta afirmación evidencia que los procesos no solo son diferentes en cada individuo, sino que va más allá y lo lleva al terreno del marketing. El mismo autor continúa afirmando de manera particular que "la mente del director de empresa (incluyendo tanto los elementos conscientes como inconscientes) y la mente del consumidor (con sus elementos conscientes e inconscientes) interactúan, formando la "mente del mercado" (Zaltman, 2004: 16-17). 
Se puede señalar que así como existe una mente individual, también existe una mente particular en cada uno de los grupos de los stakeholders (la mente de los clientes, la mente de los proveedores, la mente de los consumidores, etc.). Cada producto tiene una imagen (impresión o idea) en la mente de cada consumidor, pero existen elementos comunes que son compartidos por un grupo de personas con motivaciones e intereses afines (Braidot, 2005). Si se considera que "un análisis exhaustivo del pensamiento y la conducta del consumidor exige comprender cómo se produce la actividad mental" (Zaltman, 2004: 15), es posible que se llegue a comprender cómo se crea la imagen en la mente de los consumidores de manera individual y colectiva. Si los profesionales de marketing ya se están dando cuenta de la importancia de conocer los procesos mentales para entenderse a sí mismos y a los consumidores, los publicitarios, los comunicadores, los diseñadores, etc., también deben conocer qué pasa en la mentes y en los cerebros de los consumidores, porque cuando estén generando ideas, resolviendo problemas y pensando soluciones para las marcas, posiblemente la ruta que seguirán las estrategias deberá pasar por el filtro del cerebro y la mente de los stakeholders.

Este es el aspecto que nos interesa comprender, cómo participa la percepción en los procesos de pensamiento qué se presentan en la mente de los consumidores. Sobre este tema Prahala y Hamel explican que "independientemente de la eficacia de funcionamiento del cuerpo (la organización), éste sigue necesitando un cerebro (rumbo estratégico)" (Prahala y Hamel, 2008: 9-10). A este respecto Frith considera que: "son nuestros órganos sensoriales, los ojos, los oídos, la lengua, etc., los que procuran el vínculo entre el mundo físico y nuestra mente” (Frith, 2008: 35). Aunque otros autores como Hayes defienden que los seres humanos poseemos seis sentidos:

"También tenemos sentidos que reciben información procedente del interior de nuestro organismo: los sentidos cinestésicos, que advierten del movimiento, es decir, nos permiten sentir nuestros músculos y articulaciones" (Hayes, 1995: 17-18).

Sin embargo, Steiner y Lindenberg (2006) hablan de doce sentidos. Lo que nos lleva al siguiente aspecto, los tipos de percepción: percepción visual, percepción espacial, percepción olfativa, percepción auditiva, percepción sinestésica, percepción táctil, percepción térmica, percepción del dolor, percepción gustativa, percepción quimioestésica, percepción del equilibrio, percepción del tiempo y la percepción háptica, relacionada con el contacto físico y las sensaciones (ej. lo que una persona siente cuando estrecha la mano de otra persona).

Todos estos tipos de percepción son desconocidos en muchos directivos de empresas, sin embargo consciente o inconcientemente intentan estar presentes en algunos procesos de comunicación que van dirigidos a los stakeholders de una marca, y también lo están en cada uno de los consumidores. Lo que aquí nos interesa entender es cómo estas diferentes percepciones influyen en las estrategias y en la imagen (impresión o idea) de marcas, de manera que las empresas tengan claro que "las estrategias y teorías no son realidades en sí mismas, sólo son representaciones (o abstracciones) en la mente de las personas" (Mintzberg, Ahlstrand y Lampel, 1997: 33) y que en los diferentes stakeholders influyen muchas percep- 
ciones. Lo anterior supone que si la marca genera información en forma deficiente o incompleta, es probable que los procesos cognitivos en los consumidores funcionen de manera diferente y/o deficiente. Incluso, puede presentarse el caso que se genere una imagen de marca distorsionada o una mala impresión de marca, lo que al final termina convirtiéndose en mala reputación.

La percepción, es entonces, la fuente primaria para el conocimiento de la mente del consumidor, es la "primera operación de nuestras facultades intelectuales, y la entrada de todo conocimiento de nuestra mente” (Locke, 1970: 71). Pero las percepciones se van constituyendo en referentes que influyen en los procesos de toma de decisiones de los stakeholders frente a la marca. Para entender cómo son los procesos que se realizan en la mente de los stakeholders, es necesario aclarar tres conceptos: estructura mental, esquema mentaly proceso mental.

Las estructuras mentales son el conjunto de experiencias del medio ambiente, informaciones y emociones que ha vivido el consumidor desde su nacimiento hasta la edad adulta. Las estructuras tienen la particularidad de generar conceptos que comienzan siendo pequeños esquemas que se van modificando dependiendo de la edad y del nivel de complejidad y de abstracción que vaya desarrollando el niño a medida que crece. Las estructuras representan el proceso del pensamiento. Se puede decir que la estructura básica del conocimiento es: el esquema (Piaget \& Inhelder, 1967). Si las marcas desean generar cambios significativos en la mente de los stakeholders, lo primero que deben hacer es desarrollar estrategias que modifiquen las estructuras mentales del consumidor, teniendo presente que este cambio puede tomar mucho tiempo, incluso años.

El esquema mental, por su parte, es la unidad mínima o básica del pensamiento. Es la información relevante que el consumidor toma de situaciones específicas que le permiten, por medio de secuencias organizadas, entender lo que pasa en ellas. Piaget (1973) consideraba que el esquema se adquiría mediante el aprendizaje como una interiorización mental que permite al individuo adoptar pautas de conducta, de manera que cuando se enfrenta a situaciones similares, podía repetirlas. Esta teoría se apoya en el modelo de la adaptación biológica propuesta por el mismo Piaget donde "el modelo se desarrolla a partir de la experiencias del niño y adopta la forma de sistemas (conocidos como esquemas) que ayudan a guiar las acciones futuras, así como a dar sentido a sus experiencias" (Banyard \& Hayes, 1995, p. 205). Pero Obradors (2007) define que "los esquemas organizan la nueva información a la vez que esta información puede modificar en cierta medida el esquema general” (p. 117).

Antes de referenciar los procesos mentales es necesario hacer distinción entre proceso mental y proceso cognitivo. El proceso mental es el "conjunto de operaciones que se encargan de gestionar los conocimientos de distinta naturaleza que intervienen en la realización de una tarea” (Monedero et al., 2007, 39.). Cuando el cerebro del consumidor capta la información por medio de los sentidos los procesa mediante tres operaciones que son: clasificación de la información, comparación y selección. El proceso cognitivo es el "funcionamiento de los mecanismos mentales que se activan automá- 
ticamente cuando atendemos, comprendemos o memorizamos" (Monereo, 2007, p. 39). Los procesos mentales más relevantes son: el pensamiento, las ideas, la imaginación, los recuerdos, la memoria, las ilusiones y las emociones en general. Estos son procesos producto del funcionamiento cerebral.

Los procesos mentales son operaciones del pensamiento que actúan sobre representaciones mentales para generar nuevas acciones. Actúan como rutas particulares del ser humano que le ayudan a procesar la información que ha percibido por medio de los sentidos y transformarla en información práctica, la información nueva es comparada con la ya existente.

Lo anterior ayuda a entender cómo la reputación se construye partiendo de la construcción de estructuras mentales (experiencias encadenadas) de las cuales algunas serán más relevantes (esquemas mentales) que otras. Los procesos mentales (generadores de nuevas experiencias que influyen en las estructuras y los esquemas) son alimentados por las acciones de comunicación de la marca, cada acción posible (publicidad, comunicación, RRPP, empaque, logotipo, etc.) alimenta procesos mentales que se fijan de manera duradera en una estructura mental llegando a construir conceptos de reputación definidos e identificables por los stakeholders.

Lo anterior evidencia la complejidad de la mente del consumidor, lo que ratifica la necesidad de identificar cómo y cuáles son, cómo se forman, cómo se modifican y cómo se afianzan las estructuras mentales del consumidor con respecto a la marca. Estas estructuras mentales que pueden darse de manera individual o grupal, son fundamentales para que las percepciones de los diferentes stakeholders contribuyan al desarrollo de acciones que repercutan en la construcción de una reputación efectiva para la marca.

\section{Conclusiones y líneas futuras de investigación}

Aunque existan en la actualidad desarrollos tecnológicos importantes, avances en materia de comunicación; académicos y empresarios aún tienen un camino importante por recorrer, al menos en materia de conceptualización de algunos factores tan importantes para las marcas como son su imagen y su reputación. El hecho de que aún no se llegue a un consenso en los elementos básicos de ambos constructos posibilita un trabajo futuro importante, de manera que se vinculen investigaciones que relacionen la marca con cada uno de sus stakeholders.

La intención de relacionar elementos intangibles de las marcas (imagen y reputación) con las percepciones de los consumidores, no obedecen a un propósito de manipulación de las mentes de los consumidores, sino, y muy por el contrario, se pretende que las marcas tengan un vínculo más estrecho y transparente con los stakeholders, mediado por un diálogo ético, en el que la misión y la visión empresarial estén siempre acordes con la satisfacción de las necesidades y el desarrollo del individuo de manera sostenible. 
La publicidad y la comunicación corporativa, que se han mantenido entre la dicotomía de un vínculo con los stakeholders mediado por la razón o la emoción, tienen ahora la posibilidad de elegir la manera en que desean generar imagen y reputación. Los estudios de las percepciones de los consumidores permitirán conocer si es la emoción o lo es la razón, la vía más apropiada para estrechar los lazos entre marca y consumidor.

El conocimiento es único en cada persona, en cada consumidor y en cada grupo de stakeholders, porque las sensaciones, las emociones, los sentimientos y la forma de percibir la realidad son diferentes en cada individuo. Así como no existen dos medios ambientes iguales, ni dos emociones iguales, tampoco existen dos estructuras mentales iguales, sencillamente porque la mente es individual.

La imagen de marca y la reputación deben ser observadas como puntos de partida para impactar de manera adecuada las percepciones de los consumidores. El interés debe centrarse en generar imagen de marca beneficiada por procesos de comunicación publicitaria, con una reputación corporativa firme, basada en el conocimiento de la identidad de la marca, solventada en las buenas prácticas corporativas y en el desempeño ético de empresarios y consumidores.

El concepto de percepción viene siendo estudiado desde hace muchos siglos, solo que ahora los avances tecnológicos muestran que el consumidor tiene un potencial sin límites para imaginar y crear cosas nuevas. Sin embargo, a pesar de que la ciencia avanza rápidamente en el conocimiento del cerebro, aún queda mucho por descubrir. Lo que debe interesarle a los hombres de marketing y publicidad es la posibilidad de aprovechar los conocimientos que surgen desde disciplinas, que hasta hace pocas décadas parecían incompatibles con la publicidad, como es el caso de la filosofía, la psicología cognitiva y la neurociencia.

La percepción del consumidor es más compleja de lo que parece, pero es susceptible de ser estudiada y comprendida. El consumidor conoce la realidad de las marcas y para ello necesita de sus sentidos, pero además, toda una base de información que ha sido almacenada a lo largo de la vida influye para que la idea o la impresión que se genere de una marca pueda ser significativa, no solo en el presente, sino en el futuro inmediato.

Por lo tanto, tras analizar la manera en que los consumidores reciben y procesan la información de las organizaciones, a través de procesos de percepción, para convertirlas en imagen y reputación corporativas, queda una gran cantidad de interrogantes y, sobre todo, líneas futuras de investigación que pueden vislumbrarse en las siguientes propuestas:

- $\quad$ Sobre los diferentes aspectos que permiten diferenciar la imagen y la reputación, en especial desde las percepciones de los stakeholders y desde las comunicaciones de la identidad de las marcas.

- Sobre la forma en que la reputación corporativa actúa en la mente de los consumidores, de manera que se entienda, desde el ámbito fisiológico y psicológico, cómo se construye una forma más avanzada de imagen de marca. 
- Sobre la posibilidad de ampliar y profundizar teóricamente en el constructo del concepto de impresión de marca, teniendo como punto de partida las posturas de diversos filósofos medievales como: Alberto Magno y Tomás de Aquino, así como filósofos modernos como Spinoza, Kant, Schopenhauer, Hegel, entre otros.

- $\quad$ Sobre cómo los factores socio-culturales influyen en las percepciones de los diferentes stakeholders de la marca y de qué manera afecta el desarrollo de la estrategia en todos sus procesos (marketing, publicidad, relaciones públicas, comunicación, etc.).

\section{Referencias bibliográficas}

Aaker, D. (1996): Construir marcas poderosas. Barcelona: Gestión.

Alsop, R. (2004): The 18 immutable laws of corporate reputation. Creating, protecting \& repairing your most valuable asset. London: Kogan Page.

Argenti, P. y Forman, J. (2002): The power of corporate communication. New York: McGraw Hill.

Ávalos, C. (2010): La marca. Identidad y estrategia. Buenos Aires: La Crujía.

Banyard, P. y Hayes, N. (1995): “Pensamientos y resolución de problemas”, en Banyard, P., Cassells, A., Grenn, P. et al (eds.): Introducción a los procesos cognitivos. Barcelona: Ariel, pp. 203-235.

Batey, M. (2008): Brand Meaning. New York: Routledge.

Bauman, Z. (2010): Mundo consumo. Ética del individuo en la aldea global. Barcelona: Paidós.

Blecua, J. (1999): Diccionario general de sinónimos y antónimos. Lengua española. Barcelona: Vox.

Braidot, N. (2005): Neuromarketing. Neuroeconomía y negocios. Buenos Aires: Norte-Sur.

Brujó, G. (2010): En clave de marcas. Madrid: LID.

Camargo, P. (2009): Neuromarketing. Decodificando a mente do consumidor. Porto: IPAM.

Capriotti, P. (2009): Branding corporativo. Fundamentos para la gestión estratégica de la identidad corporativa. Santiago: Andros.

Cerviño, J. (2002): Marcas internacionales. Cómo crearlas y gestionarlas. Madrid: Pirámide.

Cuesta, U. (2004): Psicología Social Cognitiva de la Publicidad. Madrid: Fragua.

Chaves, N. (2008): La imagen corporativa. Teoría y práctica de la identificación institucional. Barcelona: Gustavo Gili.

Costa, J. (2004): La imagen de marca. Un fenómeno social. Barcelona: Paidós.

Costa, J. (2009): El DirCom hoy. Dirección y gestión de la comunicación en la nueva economía. Barcelona: Costa Punto Com. 
Doorley, J. y García, H. (2011): Reputation Management. The key to successful public relations and corporate communication. New York: Routledge.

Dowling, G. (2001): Creating corporate reputations. Identity, image, and performance. New York: Oxford University Press.

Earl, S. y Waddington, S. (2012): Brand anarchy. Managing corporate reputation. London: Bloomsbury.

Fernández, M. (2011): Comunicación y reputación en empresas e instituciones. Experiencias profesionales y propuestas prácticas. Madrid: Universitas S.A.

Fombrun, C. (1996): Reputation. Realizing value from the corporate imagen. Boston: Harvard Business School Press.

Freeman, E., Harrison, J., Wicks, A., Parmar, B. y Colle, S. (2010): Stakeholder theory. The state of the art. Cambridge: Cambridge University Press.

Frischl, J. (2002): Manual de historia de la filosofía. Barcelona: Herder.

Frith, C. (2008): Descubriendo el poder de la mente. Cómo el cerebro crea nuestro mundo mental. Madrid: Ariel.

García, J. (2011): “Identidad corporativa un concepto que pertenece a la empresa ¿diseño?, ¿estrategia?, igestión?”, en García, J. (eds.): Identidad corporativa. Consideraciones sobre el capital-imagen de la empresa. Barcelona: Fromatic Barna, pp. 93-126.

Gardner, H. (2008): Las cinco mentes del futuro. Barcelona: Paidós.

Gázquez, J. y Sánchez, M. (2004): “La identidad e imagen de marca”, en Jiménez, A. (eds.): Dirección de productos y marcas. Barcelona: UOC, pp. 55-84.

Gobé, M. (2005): Branding emocional. El nuevo paradigma para conectar las marcas emocionalmente con las personas. Barcelona: Divine Egg.

Guzmán de, M. (2001): Para pensar mejor. Desarrollo de la creatividad a través de los procesos matemáticos. Madrid: Pirámide.

Hannington, T. (2006): Cómo medir y gestionar la reputación de su empresa. Barcelona: Deusto.

Harré, R. (2002): 1.000 años de filosofía. Madrid: Taurus.

Hatch, M. y Schultz, M. (2010): Esencia de marca. Madrid: Lid.

Hayes, N. (1995): “Percepción”, en Banyard, P. et al. (eds.): Introducción a los procesos cognitivos. Barcelona: Ariel, pp. 17-86. Hax, A. y Majluf, N. (1997): Estrategias para el liderazgo competitivo. De la visión a los resultados. Buenos Aires: Granica.

Hume, D. (1984): Tratado de la naturaleza humana I. Buenos Aires: Orbis.

Hyo-Sook, K. (2011): "A reputational approach examining publics attributions on corporate social responsibility motives", Asian Journal of Communication, 21, pp. 84-101. 
Ind, N. (1992): La imagen corporativa. Estrategias para desarrollar programas de identidad eficaces. Madrid: Díaz de Santos.

James, W. (1989): Principios de psicología. México: Fondo de Cultura Económica.

Kant, I. (2006): Crítica de la razón pura. México: Taurus.

Kapferer, J. (1992): La marca, capital de la empresa. Bilbao: Deusto.

Keller, K. (2008): Administración estratégica de marca branding. México: Pearson.

Komisarjevsky, C. (2012): The power of reputation. Strengthen the asset that will make or break your career. New York: Amacom.

Landan, E. (1987): El vivir creativo. Teoría y práctica de la creatividad. Barcelona: Herder.

Lange, D., Lee, P. y Dai, Y. (2011): “Organizational reputation: a Review”, Journal of Management, 37(1), pp. 153-184.

Locke, J. (1970): Ensayo sobre el entendimiento humano. Buenos Aires: Aguilar.

López, V. y Iglesias, S. (2006): “Percepciones directivas del recurso reputación. Estudio empírico de sus relaciones con el rendimiento empresarial”, Cuadernos de Economía y Dirección de la Empresa, 28, pp. 139-160.

López, B. y Sebastián, A. (2009): “Responsabilidad Social Corporativa y reputación corporativa”, en Sánchez, J. y Pintado, T. (eds.): Imagen corporativa. Influencia en la gestión empresarial. Madrid: ESIC, pp. 139-170.

Llinás, R. (2003): El cerebro y el mito del yo. El papel de las neuronas en el pensamiento y el comportamiento humanos. Bogotá: Norma.

Martínez-Freire, P. (1995): La nueva filosofía de la mente. Barcelona: Gedisa.

Martínez, I. y Olmedo, I. (2009): “La medición de la reputación empresarial: problemática y propuesta”, Investigaciones Europeas, 15(2), pp. 127-142.

Melendo, T. (2001): Introducción a la filosofía. Pamplona: EUNSA.

Mintzberg, H., Quinn, J. y Voyer. J. (1997): El proceso estratégico. Conceptos, contextos y casos. México: Prentice Hall.

Monereo, C. (2007): Estrategias de enseñanza y aprendizaje. Formación del profesorado y aplicación en la escuela. Barcelona: Graó.

Mora, F. (2009): Cómo funciona el cerebro. Madrid: Editorial.

Nikolaeva, R. y Bicho, M. (2011): "The role of institutional and reputational factors in the voluntary adoption of corporate social responsibility reporting standards", Journal of The Academy of Marketing Science, 39(1), pp. 136-157.

Obradors, M. (2007): Creatividad y generación de ideas: Estudio de la práctica creativa en cine y publicidad. Bellaterra: Universidad Autónoma de Barcelona. 
Olcese, A., Rodríguez, M. y Alfaro, J. (2008): Manual de la empresa responsable y sostenible. Madrid: McGraw Hill.

Orozco, J. y Roca, D. (2011): “Construcción de imagen de marca y reputación a través de campañas publicitarias de RSC”, Sphera Publica, 11, pp. 273-289.

Ostberg, J. (2007): “Identidad, cultura e imagen corporativa”, en Jiménez, A. y Rodríguez, I. (eds.): Comunicación e imagen corporativa. Barcelona: UOC, pp. 15-53.

Piaget, J. y Inhelder, B. (1967): Génesis de las estructuras lógicas mentales. Clasificación y seriaciones. Buenos Aires: Guadalupe.

Piaget, J. (1973): Psicología de la inteligencia. Buenos Aires: Psique.

Piaget, J. (1994): Seis estudios de psicología. Bogotá: Labor.

Pinker, S. (2001): Cómo funciona la mente. Barcelona: Destino.

Prahalad, C. K. y Hamel, G. (2008): Estrategia en el siglo XXI. Barcelona: Deusto.

Quevedo, E. (2003): Reputación y creación de valor. Madrid: Thomson.

Ratey, J. (2002): El cerebro: manual de instrucciones. Barcelona: Mondadori.

Rey, F. y Bartoli, J. (2008): Reflexiones sobre el management de la comunicación. Buenos Aires: La Crujía.

Ruesch, J. y Bateson, G. (2009): Communication: the social matrix of psychiatry. New Jersey: Transaction.

Salinas, G. (2007): Valoración de marcas. Bilbao: Deusto.

Sanz de la Tajada, L. (1996): Auditoría de la imagen de empresa. Métodos y técnicas de estudio de la imagen. Madrid: Síntesis.

Saranyana, J. I. (1989): Historia de la filosofía medieval. Pamplona: EUNSA.

Solomon, R. (2009): El pequeño libro de filosofía. Barcelona: Ariel.

Steiner, R. y Lindenberg, C. (2006): Los doce sentidos del hombre. Buenos Aires: Antroposófica.

Sternberg, R. (1986): Las capacidades humanas. Un enfoque desde el procesamiento de la información. Barcelona: Labor.

Stuebs, M. y Sun, L. (2010): “Business reputation and labor efficiency, productivity and cost”, Journal of Business Ethics, 96(2), pp. 265-283.

Suviri, J. (2010): “Conceptualización y comparación de distintos modelos de evaluación de la reputación corporativa”, Cuadernos de Gestión del Conocimiento Empresarial, 22, pp. 179-190.

Toro, J. (2009): La marca y sus circunstancias. Vademécum de brand management. Barcelona: Deusto.

Van Riel, C. (1997): Comunicación corporativa. Madrid: Prentice Hall. 
Villafañe, J. (2004): La buena reputación. Claves del valor intangible de las empresas. Madrid: Pirámide.

Villafañe, J. (2005): La comunicación empresarial y la gestión de los intangibles en España y Latinoamérica. Madrid: Pirámide.

Zaltman, G. (2004): Cómo piensan los consumidores. Barcelona: Urano. 